\title{
Feasibility and relevance of right parasternal view for assessing severity and rate of progression of aortic valve stenosis in primary care it
}

\author{
Giovanni Benfari a,*, Anna Maria Gori ${ }^{\mathrm{b}}$, Andrea Rossi ${ }^{\mathrm{a}}$, Barbara Papesso ${ }^{\mathrm{c}}$, Corrado Vassanelli a \\ Giovanni Battista Zito ${ }^{\mathrm{d}}$, Stefano Nistri ${ }^{\mathrm{c}}$ \\ a University of Verona, Section of Cardiology, Verona, Italy \\ b Department of Medical and Surgical Critical Care, University of Florence, Florence, Italy \\ c Cardiology Service, CMSR Veneto Medica, Altavilla Vicentina, Italy \\ d Cardiology Service, ASL Napoli 3 Sud, Pompei, Italy
}

\section{A R T I C L E I N F O}

\section{Article history:}

Received 22 December 2016

Received in revised form 17 April 2017

Accepted 26 April 2017

Available online $\mathrm{xxxx}$

\section{Keywords:}

Aortic stenosis

Echocardiography

Right parasternal view

Progression rate

Primary care

\begin{abstract}
A B S T R A C T
Background: Right parasternal view (RPV) is important in assessing the severity of aortic stenosis (AS). However, the feasibility and relevance of RPV in primary care is unresolved. Moreover, information regarding the role of $\mathrm{RPV}$ in the evaluation of the hemodynamic progression of AS is lacking.

Methods: Consecutive patients with peak aortic valve velocity $\left(\mathrm{V}_{\max }\right) \geq 2.5 \mathrm{~m} / \mathrm{s}$ were prospectively enrolled in a primary care echocardiographic laboratory. Aortic Doppler parameters were evaluated from apical view and RPV. Results: The total number of enrolled patients was 330 (aged $81 \pm 11$ years, $47 \%$ female, left ventricular ejection fraction $64 \pm 9 \%$ ). The RPV was feasible in 275 (83\%). $V_{\max }$ and Mean Gradient were significantly higher and aortic valve area was significantly lower from RPV as compared to apical view $(p<0.0001$ for all). Reclassification of severity towards either moderate or severe AS occurred in $13-26 \%$ of patients, according to different criteria, when evaluated from RPV. Among 108 patients (40\%) undergoing multiple examinations the rate of progression was lower from the apical approach than from the RPV $(0.19 \pm 0.20 \mathrm{~m} / \mathrm{s} /$ year vs. $0.24 \pm 0.27 \mathrm{~m} / \mathrm{s} /$ year, respectively; $p=0.03$ ), and was fast (>0.3 m/s/year) in 17 patients (16\%) from the apical window vs. 26 patients (24\%) from RPV $(p<0.0001)$.

Conclusion: Implementing RPV is feasible in primary care and results in a substantial reclassification rate through the entire spectrum of AS severity. Our data also suggest a potential role of Doppler interrogation from multiple windows to improve AS progression assessment.
\end{abstract}

() 2017 Elsevier B.V. All rights reserved.

\section{Introduction}

Aortic valve stenosis (AS) is a common disorder which is often managed in the primary care setting during the long-term asymptomatic phase characterizing its natural history [1,2]. Decisions on management rely on the accurate assessment of stenosis severity, ventricular function and symptomatic status [3,4]. Doppler echocardiography plays a pivotal role in the non-invasive hemodynamic evaluation of these patients. The accurate evaluation of severity and progression of AS is thus needed to tailor the management, to schedule the proper follow-up strategy, and to refer for further evaluation after correct interpretation of the symptomatic status [5].

Although it has been previously emphasized that multiple acoustic windows are mandatory to properly determine the highest transvalvular

\footnotetext{
is Funding sources: none.

* Corresponding author at: University of Verona, Section of Cardiology, Piazzale Stefani n.1, 37126, Verona, Italy.

E-mail address: giovanni.benfari@gmail.com (G. Benfari).
}

velocity [6], this approach may not be systematically adopted, particularly in the non-referral, outpatients' facilities. Apical view and right parasternal view (RPV) most frequently yield the highest peak aortic valve velocity $\left(\mathrm{V}_{\max }\right)$ though, more rarely, subcostal or supra-sternal windows may be required [4,6-8]. Available data regarding the feasibility and significance of RPV, however, have been reported by tertiary referral centers, in relatively small and selected cohorts (mainly with severe AS only), resulting into different rates of feasibility (ranging from 33 to $85 \%$ ) [10-12].

Thus, the feasibility and relevance of RPV for the hemodynamic assessment of AS in a non-referral setting are unresolved. Moreover, there are no data regarding the relevance of RPV in the assessment of the rate of progression [8-12], a relevant prognostic factor in the natural history of AS in asymptomatic individuals, independently from its severity, and clinical setting $[5,13]$. Thus, this study was aimed to prospectively assess the feasibility of the RPV approach in a large group of consecutive AS outpatients with a wide range of hemodynamic severity evaluated in a primary care setting, and its impact both on the evaluation of severity and rate of hemodynamic progression. 


\section{Methods}

All consecutive outpatients referred by their general practitioners for an echocardiographic examination, which is booked through the Central Booking office to the echocardiographic laboratory of the Cardiology Service of the CMSR-Veneto Medica who presented a thickened aortic valve and a $V_{\max } \geq 2.5 \mathrm{~m} / \mathrm{s}$ were prospectively evaluated between January 2008 and December 2012. A pre-determined echocardiographic protocol for acquisition of images, storage of the data, review and measurements, performed by a board certified echo-cardiologist with more than 15 years of experience, with commercially available ultrasound systems was adopted. As previously reported [5], left ventricular (LV) volumes, and ejection fraction (EF) were measured using biplane Simpson's method. The LV mass (in grams) was calculated using the Devereux formula and indexed for body surface area (BSA). Left ventricular outflow tract (LVOT) diameter was measured in systole from the parasternal long-axis view. LVOT time-velocity integral (TVI) was recorded with pulsed Doppler from the apical five-chamber view just proximal to the valve orifice and used to calculate stroke volume (SV) which was indexed for BSA. $\mathrm{V}_{\max }$ aortic valve TVI, and transaortic Mean Gradient (MG) were measured during the same examination from the five-chamber apical view with the patients on left side decubitus, and from RPV with the patients on the right-side decubitus, by continuous wave Doppler using two different probes for each patient i.e. the combined imaging and Doppler transducer and the non-imaging dedicated continuous Doppler transducer (i.e.: pedal probe) [14] Similarly, with both combined and non-imaging probes, suprasternal and right supraclavicular approaches were used after proper positioning of the patient [13]. In each projection, the average of two to five measurements was recorded $[3,14]$. In addition, periodical evaluations for inter- and intra-observer variability were performed between the two physicians (SN, BP) practicing in the centre.

Grading of AS severity was defined based singularly either on $V_{\max }$ and MG or by aortic valve area (AVA), calculated using the continuity equation and indexed by body surface area (AVAi). The impact of RPV on each of the four grading parameters ( $V_{\max }, M G, A V A$, and AVAi) was independently evaluated.

A subanalysis of the RPV role was conducted on patients with severe AS according to $\mathrm{AVA}<1 \mathrm{~cm}^{2}$ calculated from apical 5-chamber view, preserved $\mathrm{EF}(\geq 50 \%)$, and paradoxically low MG $(\leq 40 \mathrm{~mm} \mathrm{Hg})$ and stroke volume index $\left(\leq 35 \mathrm{ml} / \mathrm{m}^{2}\right)$ [15]. The rate of progression based on $\mathrm{V}_{\max }$ was analyzed in a subgroup of patients referred for multiple echocardiographic examinations. Mean progression of aortic jet velocity (expressed as $\mathrm{m} / \mathrm{s} /$ per year) was calculated by dividing the difference between the last and first echocardiographic examination by the interval between the examinations and was graded as slow or fast according to a cut-off value of $0.3 \mathrm{~m} / \mathrm{s}$ increase per year [2,5]. Informed consent was obtained from each patient; the Internal Review Board approved the study protocol and the work has been carried out in accordance with the Code of Ethics of the World Medical Association (Declaration of Helsinki).

\subsection{Statistical analysis}

Statistical analysis was performed using the software package SPSS 22 (SPSS Inc., Chicago, Illinois). Discrete data were summarized as frequencies, and continuous data were expressed as means and standard deviations. To assess normality distributions of the measured variables a Kolmogorov-Smirnov's test was used. The $\chi^{2}$ test was used for comparison of categorical variables, and the pair or unpaired 2-tailed Student $t$-test test were used to test differences among continuous variables.

Agreement between the classification of severe stenosis by using $\mathrm{V}_{\max }$, Mean Gradient, AVA and AVA index from the apical and right parasternal windows was assessed using Cohen's Kappa (agreement: $<0.4$, pr-fair; 0.4-0.6 moderate, 0.6-0.8 good; $>0.80$, very good).

A Bland-Altman analysis was performed to assess the differences in echocardiographic parameters between the apical window and right parasternal window. The relationship between the apical window and right parasternal window was also evaluated by using Deming regression analysis.

The intraclass correlation coefficient (ICC) with $95 \%$ confidence interval (CI) was used to measure overall interobserver agreement for the echocardiographic parameters both at the apical and right parasternal windows.

\section{Results}

\subsection{Patient characteristics and feasibility of RPV}

During the study period, 330 AS patients were consecutively assessed (Table 1): 140 (47\%), were female, the vast majority (307; $93 \%$ ) showed preserved EF ( $>50 \%$ ), and no AS-related symptoms (313, 94\%). As far as cardiovascular risk profile is concerned, 247 (75\%) patients had systemic hypertension, 119 (36\%) hypercholesterolemia, 86 (23\%) diabetes, 83 (25\%) history of coronary artery disease, and 23 (7\%) were current smokers.

Apical five-chamber view approach was feasible in all patient, whereas RPV was feasible in 275 (83\%) patients. There were no differences in demographic, clinical, and echocardiographic characteristics between patients with or without RPV ( $p>0.2$ for all). In all the patients,
$\mathrm{V}_{\text {max }}$ by the non-imaging dedicated continuous Doppler transducer resulted significantly higher $(p=0.01)$ than that obtained by the usual combined imaging and Doppler transducer from the RPV $(p<0.0001)$. Overall, in 214/275 (78\%) patients $V_{\max }$ was higher when detected from RPV vs. apical view. Similarly, MG was higher from RPV vs. apical view in $219 / 275$ (80\%) of cases. In only 1 patient, $V_{\max }$ was higher from the right supraclavicular view than from any other approach (included as RPV in the subsequent analysis).

\subsection{Grading of AS severity}

On average, each criterion for AS severity was significantly different if assessed from the apical or from the RPV (Fig. 1A). The impact of RPV on severity reclassification is substantial for each of the 4 parameters across all spectra of severity, (Fig. 2): for instance, 30 patients with mild AS based on $V_{\max }$ were reclassified upward as moderate $(28 / 30$, $93 \%)$ or, more rarely, severe AS $(2 / 30,7 \%)$ from RPV, with an overall prevalence of severe AS changing from $28 \%$ to $38 \%(p<0.0001)$. Moreover, patients with 3 or 4 criteria for severe AS (i.e.: $V_{\max }>4 \mathrm{~m} / \mathrm{s}$, Mean Gradient $>40 \mathrm{~mm} \mathrm{Hg}$, AVA $<1 \mathrm{~cm}^{2}$, or AVA index $<0.6 \mathrm{~cm}^{2} / \mathrm{m}^{2}$ ) were $68 / 275$ (25\%) from the apical window and 102/275 (37\%) from the $\operatorname{RPV}(p<0.0001)$. According to this composite criterion, only one patient was classified severe by the apical window approach and not severe by the RPV, whereas 35 patients were severe by the RPV, but not by apical window.

The deltas for $\mathrm{V}_{\max }$, MG, AVA and AVAi values between the apical window and RPV were analyzed by the different severity subgroups (Table 2). No homogeneous pattern was detected across the 4 parameters.

The agreement between the apical window approach and the RPV was overall good for all the 4 criteria. After dividing patients into mild, moderate, and severe according to $\mathrm{V}_{\max }$ or $\mathrm{MG}$, the agreement between the two approaches resulted good as well for $\mathrm{V}_{\max }$ (Kappa $=0.67, p<$ 0.0001 ), and AVA (Kappa $=0.68, p<0.0001$ ), but moderate for MG (Kappa $=0.59, p<0.0001$ ). The Deming regression equation, describing the relationship between $V_{\max }$ measured by RPV and apical window, overall showed a slope of 1.11 (95\% CI 1.04 to 1.18) and an intercept of -0.17 (95\% CI -0.40 to 0.06 ) (Fig. 1B). The slope was 3.29 (95\% CI 2.24 to 5.33$)$ with an intercept of $-6.04(95 \% \mathrm{CI}-1.72$ to -3.14$)$ in patients with mild AS, whereas in the group of moderate severity the slope was 1.48 (1.27 to 1.74 ) and the intercept was -1.49 (95\% CI -2.38 to $-0.77)$. In patients with severe AS, the regression analysis showed a slope of 1.20 (95\% CI 1.04 to 1.50$)$ and an intercept of -0.70 (95\% CI -2.01 to 0.00 ). Accordingly, the Bland-Altman plot showed that the mean difference (bias) for $\mathrm{V}_{\max }$ was $0.24 \mathrm{~m} / \mathrm{s}$ (95\% CI: 0.20 to $0.28 \mathrm{~m} / \mathrm{s}$ ) between the two approaches. The Bland-Altman plots for $\mathrm{V}_{\max }$ as well as for MG, AVA and indexed AVA are displayed in Supplementary Fig. 1.

\section{Table 1}

Clinical and echocardiographic characteristics of the overall population. The displayed aortic parameters ( $\left.\mathrm{V}_{\max }, \mathrm{MG}, \mathrm{AVA}, \mathrm{AVA}-\mathrm{I}\right)$ are obtained by apical view.

\begin{tabular}{ll}
\hline Variables & All patients $(n=330)$ \\
\hline Age (years) & $81 \pm 11$ \\
Body surface area $\left(\mathrm{m}^{2}\right)$ & $1.79 \pm 0.20$ \\
Body mass index $\left(\mathrm{kg} / \mathrm{m}^{2}\right)$ & $27.6 \pm 4.9$ \\
Systolic blood pressure $(\mathrm{mm} \mathrm{Hg})$ & $145.8 \pm 19.5$ \\
Diastolic blood pressure $(\mathrm{mm} \mathrm{Hg})$ & $78.7 \pm 10.6$ \\
Heart rate $(\mathrm{bpm})$ & $71.2 \pm 12.2$ \\
Left ventricular ejection fraction $(\%)$ & $64 \pm 9$ \\
$\mathrm{~V}_{\text {max }}(\mathrm{m} / \mathrm{s})$ & $3.45 \pm 0.72$ \\
MG $(\mathrm{mm}$ Hg) & $28 \pm 14$ \\
AVA $\left(\mathrm{cm}{ }^{2}\right)$ & $1.13 \pm 0.39$ \\
AVA-index $\left(\mathrm{cm}^{2} / \mathrm{m}^{2}\right)$ & $0.63 \pm 0.21$ \\
LV mass index $\left(\mathrm{g} / \mathrm{m}^{2}\right)$ & $121 \pm 29$ \\
Stroke volume $\left(\mathrm{ml}^{2}\right)$ & $68.1 \pm 17.8$ \\
Stroke volume indexed $\left(\mathrm{ml} / \mathrm{m}^{2}\right)$ & $47.6 \pm 9.6$ \\
\hline
\end{tabular}


A.

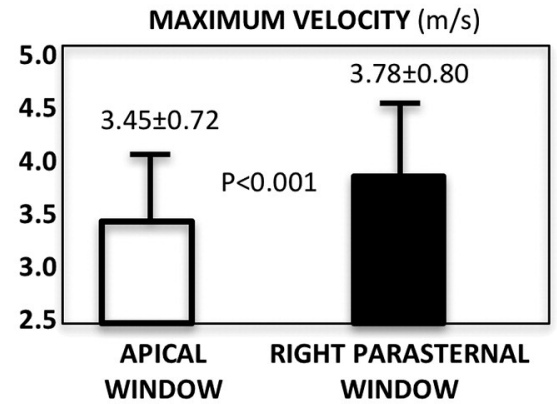

AORTIC VALVE AREA $\left(\mathrm{cm}^{2}\right)$

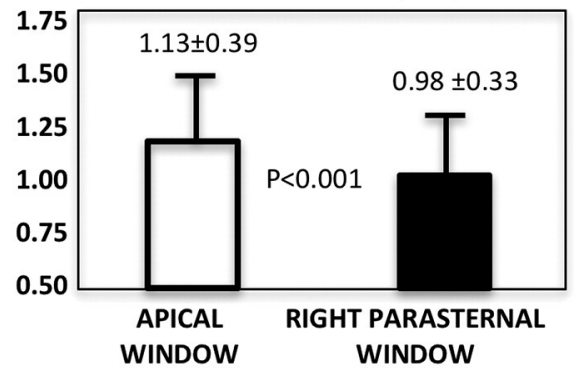

MEAN GRADIENT $(\mathrm{mmHg})$

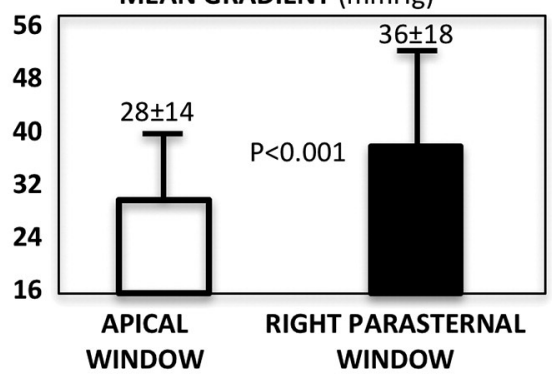

INDEXED AORTIC VALVE AREA $\left(\mathrm{cm}^{2 /} \mathrm{m}^{2}\right)$

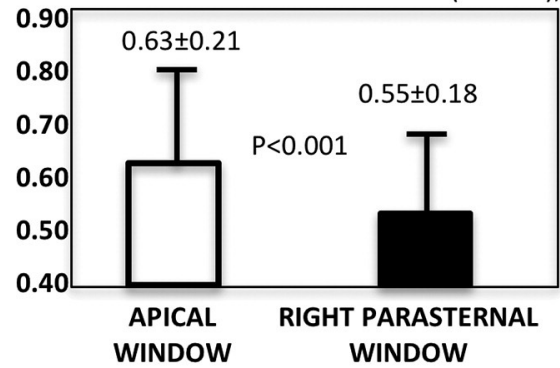

B.

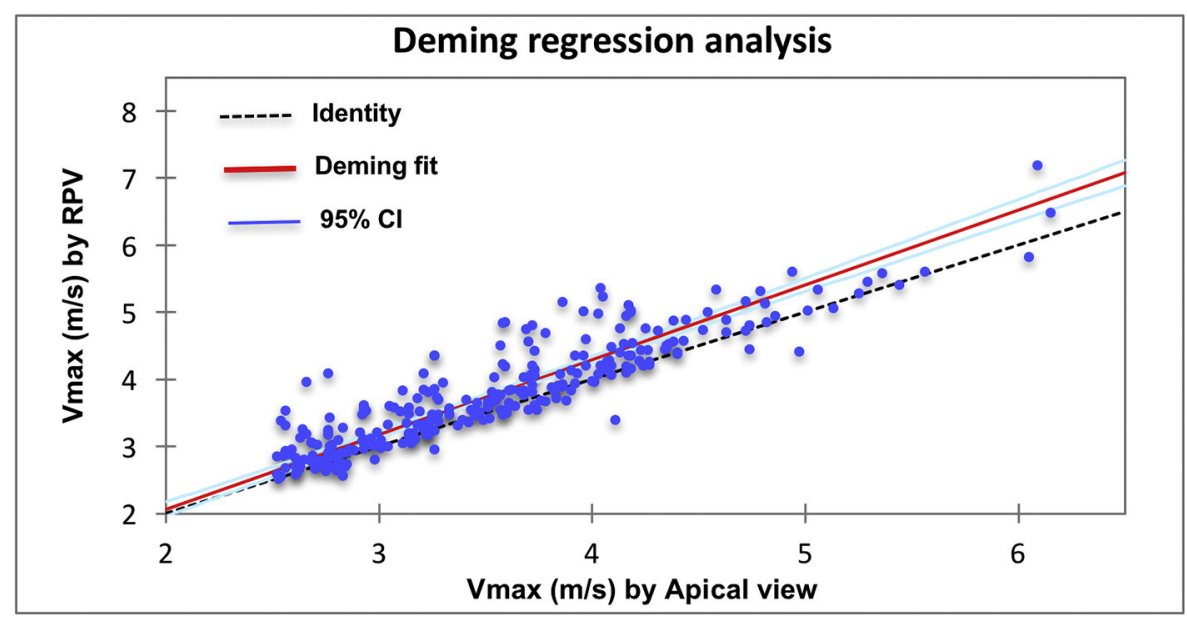

Fig. 1. A) Comparison between $V_{\max }$, MG, $A V A$ and AVA-i from apical five-chamber view and right parasternal view (RPV). $V_{\max }$ : maximal transaortic velocity, MG: mean transaortic gradient, AVA: aortic valve area by continuity equation. B) Correlation and Deming regression analysis between apical view and right parasternal view (RPV). The highlighted dot emphasizes how, even providing data that fall in the same severity grade, an accurate assessment (in this case trough RPV) is superior at the individual patient level. In this specific case, $V_{\max }$ is $3.11 \mathrm{~m} / \mathrm{s}$ from the apical view and $3.84 \mathrm{~m} / \mathrm{s}$ from RPV. $\mathrm{V}_{\max }$ : maximal transaortic velocity, MG: mean transaortic gradient, AVA: aortic valve area by continuity equation.

In 127 patients with LV ejection fraction $\geq 50 \%$ and severe AS $\left(A V A<1 \mathrm{~cm}^{2}\right)$ from the apical view, $M G<40 \mathrm{~mm} \mathrm{Hg}$ was present in $84(66 \%)$ patients of whom $13(10 \%)$ had the so-called paradoxical low-flow (stroke volume index $<35 \mathrm{ml} / \mathrm{m}^{2}$ ), low-gradient pattern while the remaining 71 patients had the normal-flow (stroke volume index $\geq 35 \mathrm{ml} / \mathrm{m}^{2}$ ) low-gradient pattern. The inconsistency between MG and AVA was significantly reduced if assessed from the RPV $[n=$ $50(39 \%) ; p<0.0001]$. In particular, inconsistent grading persisted in 8 (6\%) patients with the paradoxical low-flow low-gradient pattern and in 42 (59\%) patients with the normal-flow low-gradient pattern using the RPV ( $p<0.0001$ vs. the apical view for both), without differences between the two different flow patterns $(p=0.9)$.

\subsection{AS progression}

One hundred and eight ( $40 \%$ ) patients had $\geq 2$ echocardiographic examinations (2-9 for each patient) by both apical and RPV approach. The time between the first and last examination was $21 \pm 20$ months. The Bland Altman analysis for $\mathrm{V}_{\max }$ in overall 582 echocardiograms, thus including multiple examinations, showed similar results with respect to those obtained in 330 patients, with a mean difference (bias) for $\mathrm{V}_{\max }$ of $0.23 \mathrm{~m} / \mathrm{s}(95 \% \mathrm{CI} 0.20$ to $0.26 \mathrm{~m} / \mathrm{s})$, and the limits of agreement $-0.44 \mathrm{~m} / \mathrm{s}$ to $0.89 \mathrm{~m} / \mathrm{s}$. Similarly, the Deming regression analysis showed a slope of 1.10 (95\% CI 1.05 to 1.15) and an intercept of -0.13 ( $95 \% \mathrm{CI}-0.31$ to 0.04 ).

Overall, the rate of progression was lower from the apical view $(0.19 \pm 0.20 \mathrm{~m} / \mathrm{s} /$ year $)$ than from the RPV $(0.24 \pm 0.27 \mathrm{~m} / \mathrm{s} /$ year $p=$ 0.03 ), and was fast (i.e.: $>0.3 \mathrm{~m} / \mathrm{s} /$ year) in 17 patients $(16 \%)$ from the apical window but in 26 patients $(24 \%)$ from RPV $(p<0.0001)$.

\subsection{Reproducibility of RPV}

As far as the interobserver reproducibility is concerned, 32 patients underwent an echocardiographic examination by two physicians. The mean ICC for interobserver reproducibility was 0.998 (CI: 0.996 to $0.999, p<0.0001$ ) for $\mathrm{V}_{\max }, 0.992$ (95\% CI: 0.984 to $0.996, p<0.0001$ ) for MG and 0.995 (95\% CI: 0.991 to $0.998, p<0.0001$ ) for AVA. 


\section{Aortic valve hemodynamic assessment from either the apical view, or the apical and the RPV views ( $N=275)$}

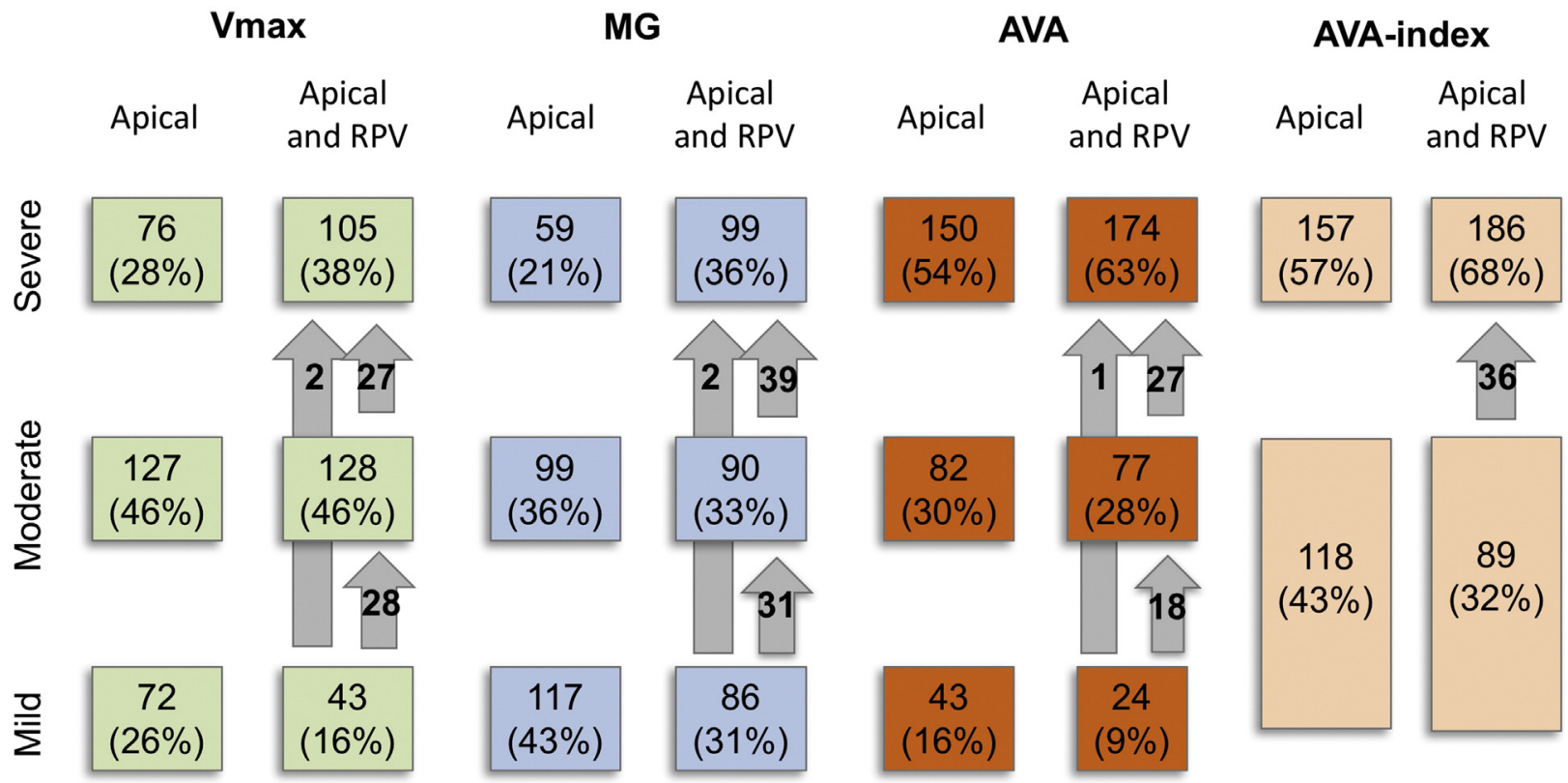

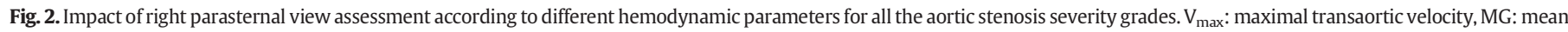
transaortic gradient, AVA: aortic valve area by continuity equation.

\section{Discussion}

Doppler echocardiography is the dominant diagnostic tool for the non-invasive hemodynamic assessment of AS. However, multiple potential technical pitfalls may affect its accuracy. In particular, improper alignment of continuous wave Doppler may result in underestimation of $\mathrm{V}_{\text {max }}$ and MG, and overestimation of AVA. To our knowledge, this is the largest study assessing the feasibility of RPV and the first to show its potential role in the evaluation of hemodynamic progression of AS. Importantly, as a further novel contribution, the present data were prospectively collected in a primary care setting, where AS patients are usually evaluated during the long asymptomatic phase of their natural history, or may be referred by their general practitioners due to changes in their symptomatic status for timely, appropriate, subsequent referral to tertiary care. Our study shows that (i) RPV is feasible in the vast majority of AS; (ii) in $>75 \%$ of the cases the highest $\mathrm{V}_{\max }$ and MG are recorded from the RPV, resulting into significant reclassification rates (1/5) across all spectra of AS severity; and (iii) in the subgroup of patients with multiple echocardiographic examinations, a faster rate of progression is more prevalent if ascertained using RPV. Furthermore, our data demonstrate that the utilization of RPV reduces the inconsistencies between MG and severely reduced AVA, in patients with either normal or reduced stroke volume index.

Other studies previously assessed the potential role of RPV in the hemodynamic assessment of AS. Overall, the feasibility of RPV varied from $33 \%$ (13) to $49 \%$ [8] in earlier studies, and up to $70-85 \%$ in the contemporary series $[9,10,11]$. Moreover, only one of the 3 latter studies was prospectively designed, and included 100 patients with any degree of severity of AS, while 1 was retrospectively designed enrolling $100 \mathrm{pa}-$ tients with severe AS referred for surgery, and the last one included 263 patients with more than moderate AS. Different from the present data, all the previous studies were performed in tertiary referral centers and none assessed the role of RPV in the evaluation of rate of hemodynamic progression of AS. Moreover, and consistently with the primary care setting in which our data were collected, our patients tended to be older, particularly those with AVA $<1 \mathrm{~cm}^{2}$ ( $83 \pm 9$ years). This may be relevant for our results, since age-related changes in aortic angulation [16] have been linked to an increased likelihood to collect higher $V_{\max }$ from the RPV. Two recent papers tried to analyze the determinants of the discrepancy between RPV and apical five-chamber view

Table 2

Differences (Delta $=$ right parasternal window - apical window) in values of $\mathrm{V}_{\max }$, Mean Gradient, AVA and AVA index according to the classification of severity.

\begin{tabular}{|c|c|c|c|c|}
\hline & \multicolumn{3}{|l|}{ Severity (apical window) } & \multirow[t]{2}{*}{$P$ for trend } \\
\hline & Mild $(n=72)$ & Moderate $(n=127)$ & Severe $(n=76)$ & \\
\hline Delta $V_{\max }(\mathrm{m} / \mathrm{s})$ & $\begin{array}{l}+0.24 \pm 0.31 \\
\text { Mild }(n=107)\end{array}$ & $\begin{array}{l}+0.29 \pm 0.34 \\
\text { Moderate }(n=98)\end{array}$ & $\begin{array}{l}+0.26 \pm 0.37 \\
\text { Severe }(n=58)\end{array}$ & 0.8 \\
\hline Delta Mean Gradient (mm Hg) & $\begin{array}{l}+3.8 \pm 5.2 \\
\text { Mild }(n=45)\end{array}$ & $\begin{array}{l}+6.3 \pm 8.6^{*} \\
\text { Moderate }(n=81)\end{array}$ & $\begin{array}{l}+7.0 \pm 8.9^{*} \\
\text { Severe }(n=149)\end{array}$ & 0.01 \\
\hline Delta AVA $\left(\mathrm{cm}^{2}\right)$ & $\begin{array}{l}-0.17 \pm 0.20 \\
\text { Non-severe }(n=117)\end{array}$ & $-0.09 \pm 0.14^{*}$ & $\begin{array}{l}-0.06 \pm 0.10^{* *} \\
\text { Severe }(n=156)\end{array}$ & 0.02 \\
\hline Delta AVA index $\left(\mathrm{cm}^{2} / \mathrm{m}^{2}\right)$ & $-0.09 \pm 0.10$ & & $-0.04 \pm 0.06$ & 0.01 \\
\hline
\end{tabular}

$* p<0.05$ vs mild.

** $p=0.001$ vs moderate. 
$[9,10]$ : although their findings indicate that the RPV is influenced by the aortic root angulation the strength of this relationship is not such that patients with a larger anteroseptal angle are excluded from the benefits of the more accurate hemodynamic definition obtained by systematic utilization of RPV [9-11]. Thus, our data demonstrate that extensive application of guidelines [6] in a non-referral facility is feasible (83\%) affording a level of efficiency which is comparable to that reported in tertiary centers.

In our study we used the Deming regression analyses to estimate the agreement between the two approaches (apical window vs parasternal approach) and possible systematic bias between them. In patients with mild and moderate AS the regression analyses showed a scarce agreement between the apical approach and parasternal approach, with significant underestimation of $\mathrm{V}_{\max }$ from the apical window with respect to the right parasternal approach. As concerned patients with severe $A S$, we found that the apical window approach underestimated $V_{\max }$ by $20 \%$ compared with the RPV approach. Therefore, the analysis of the regression equation revealed a constant [regression line's intercept] and proportional [regression line's slope] difference between the two methods showing that systematic utilization of RPV provides a more accurate measurement of the $V_{\text {max }}$. Its additional diagnostic value in patients with $A S$ is reinforced, whatever parameter $\left(V_{\text {max }}, M G, A V A\right.$ or AVAi) is taken into consideration, with $17 \%$ to $22 \%$ ( 57 to 72 ) of patients being reclassified to a higher severity grade, particularly if non-imaging probes are utilized [17]. The clinical relevance of these finding are immediate beyond grading on a single patient basis (Fig. 1B). Moreover, in asymptomatic AS patients with normal LV ejection fraction and at least one echocardiographic criterion for severe AS, the positivity for multiple criteria has been shown to have a significant additive effect compared to any single criterion alone, in predicting worse event-free survival. On the other side, a recent study of a large population of mostly symptomatic patients with AS, showed that patients with an AVA comprised between $0.8 \mathrm{~cm}^{2}$ and $1.0 \mathrm{~cm}^{2}$ exhibit a significant improvement in survival with isolated AVR [19]. Each of these features, either alone or in combination, were significantly more prevalent if examined from the RPV than from the apical view in the present cohort.

Also, the role of RPV has been proposed as relevant in the challenging group of patients with discordant echocardiographic grading of stenosis severity and normal ejection fraction [11,17]. Our findings show that the systematic adoption of RPV results in a 44\% reconciliation of the overall discrepancy between severely reduced AVA $\left(<1 \mathrm{~cm}^{2}\right)$ and only moderately ( $<40 \mathrm{~mm} \mathrm{Hg}$ ) increased MG, maintaining a comparable effectiveness in reducing this discordancy both in patients with low-flow and in those with normal-flow pattern $[11,12]$. Current guidelines [4] underscore that the first diagnostic step in patients with severely reduced AVA and discordant $\mathrm{V}_{\text {max }} / \mathrm{MG}$ is to ensure that data have been recorded and measured correctly. This process may be particularly relevant in outpatient facilities to avoid improper referral to other different approaches aimed at corroborating stenosis severity, usually not available in the primary care setting [17]. Such an improvement in hemodynamic definition further underscores the need for accurate associated assessment of the etiology of symptoms if AS is only moderate [20] and reinforces the complexity and difficulties of interpreting symptoms, or their lack thereof, in a population that is becoming increasingly older, deconditioned and comorbid [21], prone both to down-regulate their activity level to avert symptoms [3,4] and not to be referred to provocative or advanced imaging modalities just based on age [22]

Finally, our data also demonstrate for the first time that RPV positively affects the evaluation of the rate of hemodynamic progression of AS. Multiple studies from referral centers have provided insights into the prevalence and prognostic relevance of rapid hemodynamic progression in patients with severe, and less than severe AS [10-12]. More recently, it has been demonstrated that, in primary care, the annual rate of progression of AS is frequently very rapid, and is an independent predictor of overall mortality and AVR [5]. The present data demonstrate the clinical relevance of the non-apical approach for tailoring follow-up strategies not simply based on baseline severity but also on rate of progression, supporting the need of adding the approach from which higher $V_{\max }$ and $M G$ have been recorded on the echocardiographic report [6]. However, the incompleteness of our follow-up precludes any definitive conclusion on the value of the RPV in studying the progression of AS, prompting the need of future studies aimed to prospectively study this specific issue.

\section{Conclusion}

This is the first study assessing applicability of current echocardiographic guidelines in assessing AS in primary care. Our data demonstrate that implementing RPV is feasible in the largest study cohort purposely described to date, spanning all spectra of AS severity. Right parasternal approach, particularly if the non-imaging probe is used, implicates a consistent upgrading of $\mathrm{V}_{\max }, \mathrm{MG}$, and AVA values resulting into a significant reclassification rate. Furthermore, when applying RPV in follow-up studies, a more rapid rate of progression might be detectable. These findings may have important clinical implications when implemented in the comprehensive evaluation and follow-up evaluation of AS patients and their symptoms.

Supplementary data to this article can be found online at http://dx. doi.org/10.1016/j.ijcard.2017.04.091.

\section{Conflict of interest}

The authors report no relationships that could be construed as a conflict of interest.

\section{Acknowledgements}

All the contributors are listed as co-authors. The paper is endorsed by ARCA (Associazioni Regionali dei Cardiologi Ambulatoriali).

\section{References}

[1] S. Coffey, B.J. Cairns, B. Iung, The modern epidemiology of heart valve disease, Heart 102 (1) (2016) 75-85.

[2] R. Rosenhek, T. Binder, G. Porenta, I. Lang, G. Christ, M. Schemper, et al., Predictors of outcome in severe, asymptomatic aortic stenosis, N. Engl. J. Med. 343 (9) (2000) 611-617.

[3] B.R. Lindman, R.O. Bonow, C.M. Otto, Current management of calcific aortic stenosis, Circ. Res. 113 (2) (2013) 223-237.

[4] R.A. Nishimura, C.M. Otto, R.O. Bonow, B.A. Carabello, J.P. Erwin III, R.A. Guyton, et al., 2014 AHA/ACC guideline for the management of patients with valvular heart disease: a report of the American College of Cardiology/American Heart Association Task Force on Practice Guidelines, J. Am. Coll. Cardiol. 63 (22) (2014) e57-185.

[5] S. Nistri, P. Faggiano, I. Olivotto, B. Papesso, T. Bordonali, G. Vescovo, et al., Hemodynamic progression and outcome of asymptomatic aortic stenosis in primary care, Am. J. Cardiol. 109 (5) (2012) 718-723.

[6] H. Baumgartner, J. Hung, J. Bermejo, J.B. Chambers, A. Evangelista, B.P. Griffin, et al., Echocardiographic assessment of valve stenosis: EAE/ASE recommendations for clinical practice, J. Am. Soc. Echocardiogr. 22 (1) (2009) 1-23 (quiz 101-2).

[7] D. Corsi, A. Imperatori, A. Fontana, P. Russo, G. Trocino, Assessment of aortic stenosis from the right parasternal view: when the answer is just on the opposite side, J. Cardiovasc. Med. (Hagerstown) 14 (1) (2013) 78-80.

[8] G.A. Williams, A.J. Labovitz, J.G. Nelson, H.L. Kennedy, Value of multiple echocardiographic views in the evaluation of aortic stenosis in adults by continuous-wave Doppler, Am. J. Cardiol. 55 (4) (1985) 445-449.

[9] T. Tavli, A. Ammar, M. Wong, Doppler-derived aortic valve gradients: imaging versus non-imaging techniques, J. Heart Valve Dis. 2 (3) (1993) 253-256.

[10] C.C. de Monchy, L. Lepage, I. Boutron, M. Leye, D. Detaint, F. Hyafil, et al., Usefulness of the right parasternal view and non-imaging continuous-wave Doppler transducer for the evaluation of the severity of aortic stenosis in the modern area, Eur. J. Echocardiogr. 10 (3) (2009) 420-424.

[11] J. Thaden, V.T. Nkomo, KJ. Lee, J.K. Oh, Doppler imaging in aortic stenosis: the importance of the nonapical imaging windows to determine severity in a contemporary cohort, J. Am. Soc. Echocardiogr. 28 (7) (2015) 780-785.

[12] E.J. Cho, S.M. Kim, S.J. Park, S.C. Lee, S.W. Park, Identification of factors that predict whether the right parasternal view is required for accurate evaluation of aortic stenosis severity, Echocardiography 33 (6) (2016) 830-837.

[13] L. Hatle, B.A. Angelsen, A. Tromsdal, Non-invasive assessment of aortic stenosis by Doppler ultrasound, Br. Heart J. 43 (3) (1980) 284-292. 
[14] S. Nistri, M. Galderisi, P. Faggiano, F. Antonini-Canterin, G. Ansalone, F.L. Dini, et al., Practical echocardiography in aortic valve stenosis, J. Cardiovasc. Med. (Hagerstown) 9 (7) (2008) 653-665.

[15] P. Pibarot, J.G. Dumesnil, Low-flow, low-gradient aortic stenosis with normal and depressed left ventricular ejection fraction, J. Am. Coll. Cardiol. 60 (19) (2012) 1845-1853.

[16] O. Mirea, F. Maffessanti, P. Gripari, G. Tamborini, M. Muratori, L. Fusini, et al., Effects of aging and body size on proximal and ascending aorta and aortic arch: inner edgeto-inner edge reference values in a large adult population by two-dimensional transthoracic echocardiography, J. Am. Soc. Echocardiogr. 26 (4) (2013) 419-427.

[17] M.A. Clavel, J. Magne, P. Pibarot, Low-gradient aortic stenosis, Eur. Heart J. (2016).
[19] M. Berthelot-Richer, P. Pibarot, R. Capoulade, J.G. Dumesnil, A. Dahou, C. Thebault, et al., Discordant grading of aortic stenosis severity: echocardiographic predictors of survival benefit associated with aortic valve replacement, JACC Cardiovasc. Imaging 9 (7) (2016) 797-805.

[20] R. Rajani, J. Hancock, J.B. Chambers, The art of assessing aortic stenosis, Heart 98 (Suppl. 4) (2012) iv14-iv22.

[21] P. Faggiano, S. Frattini, V. Zilioli, A. Rossi, S. Nistri, F.L. Dini, et al., Prevalence of comorbidities and associated cardiac diseases in patients with valve aortic stenosis Potential implications for the decision-making process, Int. J. Cardiol. 159 (2) (2012) 94-99.

[22] A. Bowling, Ageism in cardiology, BMJ 319 (7221) (1999) 1353-1355. 